\title{
Enhanced tumour antiangiogenic effects when combining gefitinib with the antivascular agent ZD6126
}

\author{
A Bozec', S Lassalle', J Gugenheim², J-L Fischel', P Formento', P Hofman² and G Milano*,I \\ 'Oncopharmacology Unit, Centre Antoine-Lacassagne, 33, Avenue de Valombrose, 06189 Nice Cedex 2, France; ${ }^{2}$ General Hospital, Nice, France
}

\begin{abstract}
Current experimental and clinical knowledge supports the optimisation of endothelial cell targeting using a strategy combining antiEGFR drugs with antivascular agents. The purpose of the present study was to examine the effects of the association of ZD6 I 26, an antivascular microtubule-destabilising agent, with gefitinib and irradiation on the growth of six head and neck human cancer cell lines xenografted in nude mice and to study predictive and molecular factors responsible for antitumour effects. CAL33- and Hep-2grafted cell lines were the most sensitive to ZD6I26 treatment, with VEGF levels significantly higher $(P=0.0336)$ in these tumour xenografts compared to Detroit 562- and CAL27-grafted cell lines with relatively low VEGF levels that were not sensitive to ZD6I26. In contrast, neither IL8 levels nor EGFR expression was linked to the antitumour effects of ZD6I26. ZD6I26 in combination with gefitinib resulted in a synergistic cytotoxic interaction with greater antitumour effects than gefitinib alone. The synergistic interaction between ZD6I26 and gefitinib was corroborated by a significant decrease in CD3I labelling. The present study may serve for future innovative clinical applications, as it suggests that VEGF tumour levels are possible predictors for ZD6 I 26 antitumour efficacy. It also supports the notion of antitumour supra-additivity when combining gefitinib and ZD6126, and identifies neoangiogenesis as the main determinant of this synergistic combination.

British Journal of Cancer (2006) 95, 722-728. doi: I0.1038/sj.bjc.6603308 www.bjcancer.com
\end{abstract}

Published online 29 August 2006

(c) 2006 Cancer Research UK

Keywords: EGFR targeting; antivascular agents; gefitinib; ZD6126

Tumour expression of growth factor receptors of the HER family makes it possible to perform innovative therapeutic targeting as demonstrated by the development of clinically active drugs such as herceptin $\left({ }^{\mathrm{B}}\right)$, cetuximab and EGFR tyrosine kinase inhibitors (Gross et al, 2004). The antiproliferative and antitumour effects of these compounds are attributed to cellular physiological consequences following receptor targeting manifested by inhibition of cell proliferation, increased apoptosis and reduced tumour neoangiogenesis (Woodburn, 1999). This ability to reduce tumour neoangiogenesis by EGFR targeting has been attributed to the inhibition of proangiogenic tumour factors such as VEGF and FGF (Perrotte et al, 1999; Ciardiello et al, 2001). An interesting recent study points out that EGFR targeting of tumours can also include the endothelial cell network (Hirata et al, 2002). Endothelial cells express EGFR. Thus, part of the global antiangiogenic effect resulting from EGFR targeting can also be attributed to the direct impact of EGFR-targeting agents on endothelial cells. Consequently, a variable part of the antiangiogenic activity of EGFRtargeting drugs could be caused by a dual effect: inhibition of proangiogenic factors such as VEGF produced by the tumour itself and a direct effect on the intra-tumour endothelial cells. It would be interesting therefore to optimise endothelial cell targeting using a strategy combining anti-EGFR treatment and an antivascular approach. The vascular-targeting compound ZD6126 destabilises microtubules and selectively disrupts immature tumour endothelial cells, which stops tumour blood flow and induces tumour cell

*Correspondence: Dr G Milano; E-mail: gerard.milano@nice.fnclcc.fr Received 22 June 2006; revised 7 July 2006; accepted 13 July 2006; published online 29 August 2006 death (Thorpe et al, 2003). A recent phase I study with ZD6126 underlines the potential impact of this compound on the vasculature (Beerepoot et al, 2006). Associating ZD6126 with the EGFR tyrosine kinase inhibitor gefitinib would thus combine an antivascular agent and an anti-EGFR drug. Furthermore, as ZD6126 is a compound acting on microtubules, it cannot be excluded that part of its antitumour activity may also consist of direct effects on tumour cells. Combining ZD6126 and gefitinib would thus provide a potentially beneficial association of two drugs producing optimal effects on both endothelial and tumour cells. Recent preclinical studies suggest that radiotherapy in combination with antiangiogenic/vasculature-targeting agents may enhance the therapeutic ratio of ionising radiation alone (Wachsberger et al, 2005). Thus, the association of ZD6126 and gefitinib with irradiation could also be an interesting area for investigation.

Tumour vasculature is a key target in the treatment of solid tumours, particularly of the head and neck (Le and Giaccia, 2003). In the present study, a panel of six different head and neck human cancer cell lines was examined in a xenograft mouse model. These tumour xenografts, in athymic nude mice, were studied for predictive and molecular factors responsible for antitumour effects.

\section{MATERIALS AND METHODS}

\section{Chemicals}

Gefitinib and ZD6126 were kindly provided by AstraZeneca (Macclesfield, United Kingdom). Working solutions were prepared extemporaneously as follows: gefitinib was suspended in $0.9 \%$ 
$\mathrm{NaCl}, 0.01 \%$ Tween-80 and ZD6126 in $0.9 \% \mathrm{NaCl}$ with $\mathrm{pH}$ adjusted to 4.5. For both drugs, the concentrations were adjusted so as to include the daily dose in $0.2 \mathrm{ml}$ of drug suspension. Dulbecco's modified Eagle's medium (DMEM), penicillin, streptomycin and glutamine were purchased from Whittaker (Verviers, Belgium). Foetal bovine serum (FBS) was obtained from Dutscher (Brumath, France).

\section{Cell lines}

Six different human head and neck cancer cell lines: CAL27, CAL33, CAL60 and CAL166 originated from our institution (the Centre Antoine Lacassagne); Detroit 562 and Hep-2 were obtained from the American Type Culture Collection (Rockville, MD, USA). These cell lines exhibit variable EGFR levels (ligand-binding assay; Dassonville et al, 1993), doubling time, in vitro sensitivities to gefitinib or RT and p53 status, as summarised in Table 1.

The cell lines were maintained as monolayer cultures in DMEM supplemented with $10 \%$ FBS $\left(\mathrm{v} \mathrm{v}^{-1}\right), 2 \mathrm{~mm}$ glutamic acid, $50000 \mathrm{Ul}^{-1}$ penicillin and $80 \mu \mathrm{M}$ streptomycin in a humidified incubator (Sanyo, Japan) at $37^{\circ} \mathrm{C}$ in an atmosphere containing $8 \%$ $\mathrm{CO}_{2}$. Batches of $15 \times 10^{6}$ cells were frozen in FBS supplemented with $5 \%$ DMSO $\left(\mathrm{vv}^{-1}\right)$ in advance for injection into mice. Shortly before injection, cells were thawed and suspended in Ringer lactate.

\section{Mice}

Animal experiments were performed in accordance with the regulations of the institutional ethical commission and of the United Kingdom Co-ordinating Committee on Cancer Research guidelines (Workman et al, 1998). Six-week-old female Swiss nude mice were purchased from Charles River (L'Arbresle, France) and received subcutaneous inoculation in the right flank of $2 \times 10^{6}$ cells dissolved in $100 \mu \mathrm{l}$ of Ringer lactate (six to eight animals per treatment condition).

\section{Tumours}

Tumour length and width were measured weekly using a caliper. Tumour volume was calculated as $\pi / 6 \times$ length $\times$ width $^{2}$ until animal killing. At that time, animals were killed by spinal cord dislocation and tumours were subsequently removed surgically and weighed; half of the tumour was directly frozen in liquid nitrogen for protein analysis and the other half fixed in paraformaldehyde overnight for CD31 and Ki67 examination using microtissue array (MTA immunochemistry).

\section{Preparation of samples for analysis of cellular factors}

Frozen tumours were pulverised in a liquid nitrogen-cooled Thermovac pulveriser. The resulting powders were homogenised in 10 volumes of a $10 \mathrm{~mm}$ Tris- $\mathrm{HCl}$ buffer ( $\mathrm{pH} 7.4$ ), containing $1 \mathrm{~mm}$ EDTA, $0.5 \mathrm{~mm}$ dithiothreitol and $10 \mathrm{~mm}$ sodium molybdate.
The homogenates were centrifuged for $1 \mathrm{~h}$ at $105000 \mathrm{~g}\left(+4^{\circ} \mathrm{C}\right)$ and the supernatants (cytosols) were used for protein determination by immunoblotting. Total protein content was measured using the bicinchoninic acid assay.

VEGF and IL8 were determined by ELISA using DVE 00 and D8050, respectively, from Quantikine (Minneapolis, USA); EGFR was determined by the ${ }^{125}$ I-EGF binding method followed by Scatchard-plot analysis. These factors were chosen because VEGF is the main growth factor for endothelial cells; IL8 is a proangiogenic factor (as VEGF, bFGF and aFGF) with a proven role in different tumour types and EGFR as it is the target of gefitinib.

The microvessel marker CD31 (DAKO monoclonal antibody M/ B1 ref.: M7240) and the proliferation marker Ki67 (DAKO monoclonal antibody JC70A ref.: M0823) were determined by MTA on treated tumours obtained after animal killing.

\section{Treatment}

In vivo growth characteristics of the human head and neck cancer xenografts Tumour volume was monitored once a week between 3 and 8 weeks depending on the cell line; the experiments were stopped when tumours reached $1 \mathrm{~cm}^{3}$. The differential expression of molecular factors potentially related to ZD6126 sensitivity such as VEGF, IL8 (by ELISA) and EGFR (by ligand-binding assay) was measured, at the end of the observation period, in untreated tumours (tumour volume between 72 and $2344 \mathrm{~mm}^{3}$ ) after animal killing.

Sensitivity of the six cell lines to ZD6126 Treatment was applied when tumours reached a mean volume of $250 \mathrm{~mm}^{3}$; animals were treated once a week for 3 weeks, with freshly prepared ZD6126 $\left(200 \mathrm{mg} \mathrm{kg}^{-1}\right)$ (Goto et al, 2004), or vehicle only (controls), and the tumour volume monitored for 2 weeks after the end of treatment period or until the mean volume of the controls reached $1 \mathrm{~cm}^{3}$. Mice injected with CAL166 and CAL33 tumours, which grow relatively quickly, could only be treated twice whereas all other mice models were treated three times.

Effects of a single drug and their combination with irradiation on tumour growth, tumour vessel density and molecular factors representative of proliferation The effects of each single drug and their combination with or without irradiation (RT) were examined for the impact on tumour growth and tumour angiogenesis (CD31) and on molecular factors indicative of proliferation (Ki67). CD31 and Ki67 expressions were determined in MTAs by immunohistochemistry (Simon et al, 2004) on CAL33, a highly proliferative and moderately responsive cell line. The final score was the result of the examination of three fields per tumour, and between four and eight tumours were investigated. Labelling intensities were scored as $0=$ no, $1=$ slight, $2=$ medium, $3=$ strong and $4=$ very strong. Scoring was performed at the end of the treatment sequence, on day 15 , and at the end of

Table I Characteristics of head and neck cancer cell lines (historical data from in vitro experiments Magné et al, 2002)

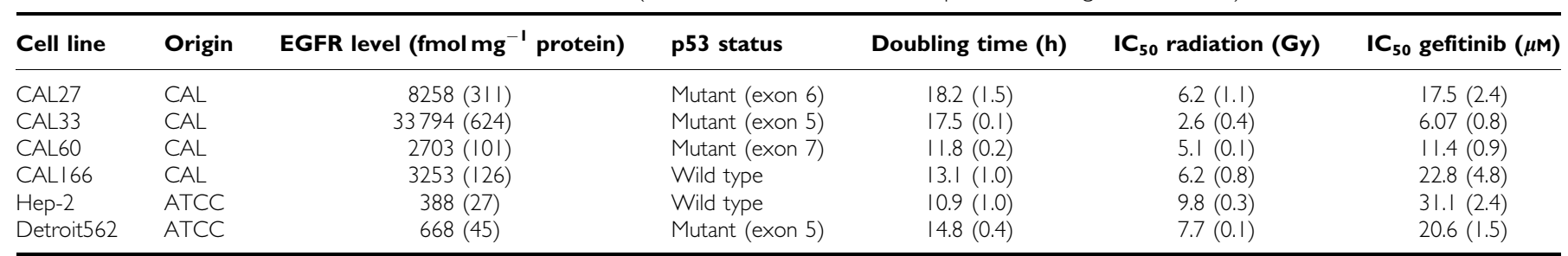

ATCC = American Type Culture Collection, Rockville; CAL = Centre Antoine Lacassagne; EGFR=epidermal growth factor receptor. Mean values (standard deviation) for EGFR levels, doubling time and $I_{50}$ values. At least two separate experiments were performed to evaluate the EGFR content, and three individual experiments for the other determinations. 
experiments on day 33. Time of termination of experiments was dictated by ethical reasons (tumour volume in controls).

Mice bearing well-established CAL33 tumours (mean tumour volume/treatment group $\sim 250 \mathrm{~mm}^{3}$ ) were treated every week with vehicle alone (controls), ZD6126 (150 $\mathrm{mg} \mathrm{kg}^{-1}, 0.2 \mathrm{ml}$ intraperitoneally on days 0,7 and 14$)$, gefitinib $\left(120 \mathrm{mg} \mathrm{kg}^{-1}\right.$ day $^{-1}, 5$ days week $^{-1}$ during 2 weeks, $0.2 \mathrm{ml} \mathrm{os}^{-1}$ ) and RT (3 Gy day ${ }^{-1}, 3$ days week $^{-1}$ during 2 weeks). The dose of gefitinib was chosen according to previous data in tumour xenografts in mice (Formento et al, 2005) and the dose of ZD6126 was diminished when compared to the monotherapy study in order to allow the interaction with other agents to be put into evidence.

Interaction between ZD6126 and gefitinib alone or in combination with RT (when given on the same day, ZD6126 was applied $2 \mathrm{~h}$ after RT) was evaluated for tumour growth inhibition. The sequence between gefitinib and RT was already established by us from tumour experiments with the two agents applied to the same tumour cell line xenograft (Formento et al, 2005).

The effects of the treatments were evaluated as described previously (Prewett et al, 2002). Evaluation of the tumour effect consisted in measuring the mean tumour volume on different given days for the different treatment groups: controls, treatment $\mathrm{a}$, treatment $\mathrm{b}$ and treatment $\mathrm{a}+\mathrm{b}$. Fractional tumour volume (FTV) for each treatment group was calculated as the ratio between the mean tumour volumes of treated and untreated tumours. This was performed for treatment a (FTVa), for treatment $b$ (FTVb) and for treatment $a+b(F T V a+b)$. The expected FTV for the ' $a+b$ ' combination was defined as FTVa (observed) $\times$ FTVb (observed). The ratio $\mathrm{FTVa}+\mathrm{b}$ (expected)/FTVa $+\mathrm{b}$ (observed) was the combination ratio (CR). If CR $>1$, there are supra-additive effects and if $\mathrm{CR}<1$ infra-additive ones. Strictly additive effects are observed if $\mathrm{CR}=1$.

The effects on CD31 and Ki67 were evaluated using the nonparametric analysis of variance (Kruskal-Wallis test). The differences between cell lines for molecular factors (VEGF, IL8 and EGFR) were examined using the Mann-Whitney test.

\section{RESULTS}

\section{Tumour characteristics}

The fastest growing xenografts were CAL166 and CAL33 with a tumour volume doubling time of 7 and 10 days, respectively, followed by Detroit 562 (tumour volume doubling time of 15 days) (Figure 1). The other cell lines (CAL60, Hep-2 and CAL27) grew relatively more slowly under the conditions of the present study.
There was a marked variation in VEGF and IL8 levels between the six xenograft models examined. The variability in EGFR expression was smaller between the studied cell lines (Figure 2).

The tumour volume did not influence VEGF secretion (Figure 3); tumours originating from the same cell line, whatever their respective volumes, maintain very comparable VEGF levels. The VEGF intra-tumour concentration could thus be considered to be strictly cell line dependent.

\section{Effects of single-agent ZD6126}

ZD6126 on its own exhibited relatively modest tumour growth inhibition. There were marked disparities in the antitumour activity of ZD6126 between the different xenografts (Figure 4). Of note, for Detroit and CAL27 tumour xenografts, treatment with ZD6126 conferred a growth advantage. CAL33 was moderately sensitive and Hep-2 was the most sensitive to ZD6126 treatment. VEGF levels were significantly higher $(P=0.0336)$ in these tumour xenografts as compared to Detroit 562 and CAL27 xenografts for which ZD6126 increased the tumour growth (Figure 2). Neither EGFR nor IL8 tumour levels were linked to the differential sensitivity to ZD6126.

\section{Effects of ZD6126 in combination with gefitinib and radiation}

Figure 5A shows that, although ZD6126 had no apparent effect on CAL33 tumour growth, its combination with gefitinib resulted in a greater antitumour effect than with gefitinib alone $(\mathrm{CR}=1.47,1.35$ and 1.15 at 19, 26 and 33 days, respectively, after cell injection,

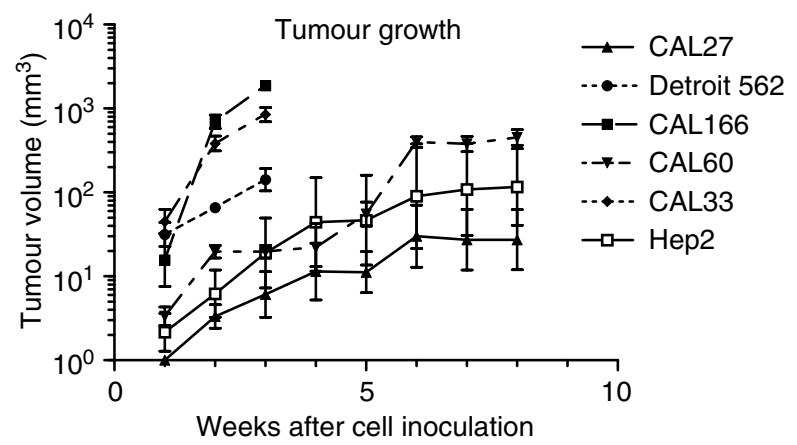

Figure I Growth (log scale) of the different xenografted tumours in athymic nude mice. Bars represent standard error of the mean.
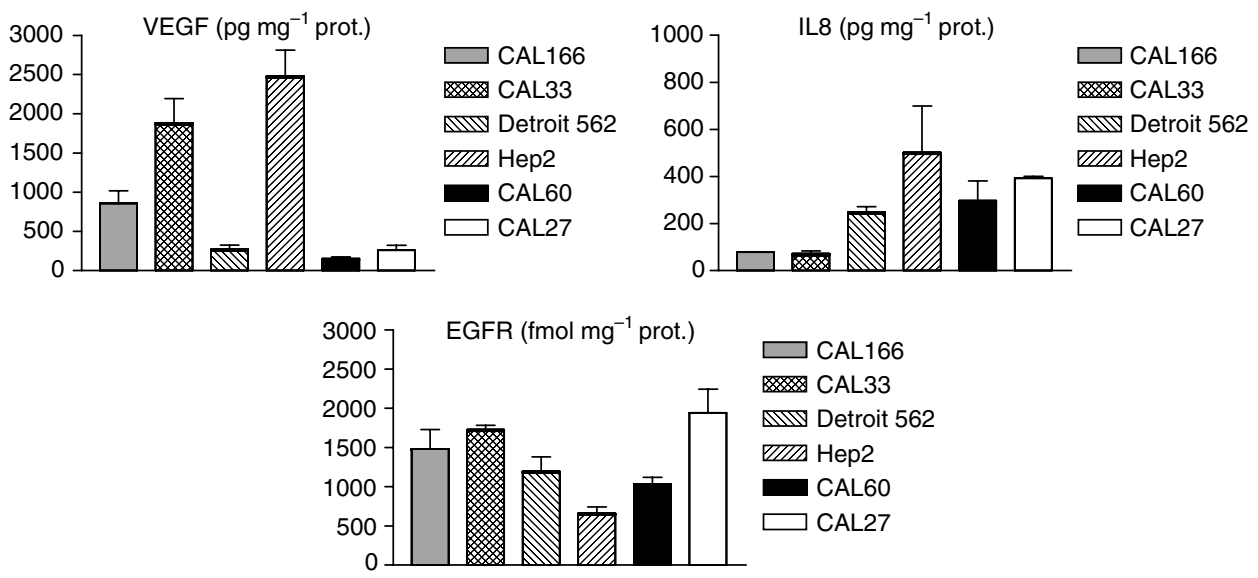

Figure 2 Distribution of VEGF ( $\mathrm{pg} \mathrm{mg}^{-1}$ protein), IL8 ( $\mathrm{pg} \mathrm{mg}^{-1}$ protein) and EGFR ( $\mathrm{fmol} \mathrm{mg}^{-1}$ protein) levels in different xenografted tumours in athymic nude mice. Bars represent standard error of the mean. 
with between five and eight mice per treatment group). The addition of RT did not modify markedly the observed antitumour effects resulting from the ZD6126 + gefitinib combination with a tendency in this case to infra-additive interaction $(\mathrm{CR}=0.87,0.85$ and 0.64 at 19, 26 and 33 days, respectively, after cell injection) (Figure 5B).

\section{Molecular parameters}

At the end of the experiment (day 33), CAL33 tumours were analysed for CD31 and Ki67 staining. Single-drug treatments with either gefitinib or ZD6126 had a modest effect on CD31 tumour labelling as compared to controls without drug (Figure 6). CD31 tumour labelling reflected accurately the antitumour effect resulting from the different combinations. Thus, the synergistic interaction between ZD6126 and gefitinib was confirmed by a significant decrease in CD31 labelling $(P<0.01)$ (Figure 6). There was a less intense impact on CD31 labelling, corroborating the effects on tumour growth, when applying the triple combination ZD6126 + gefitinib + RT as compared to the two-drug association

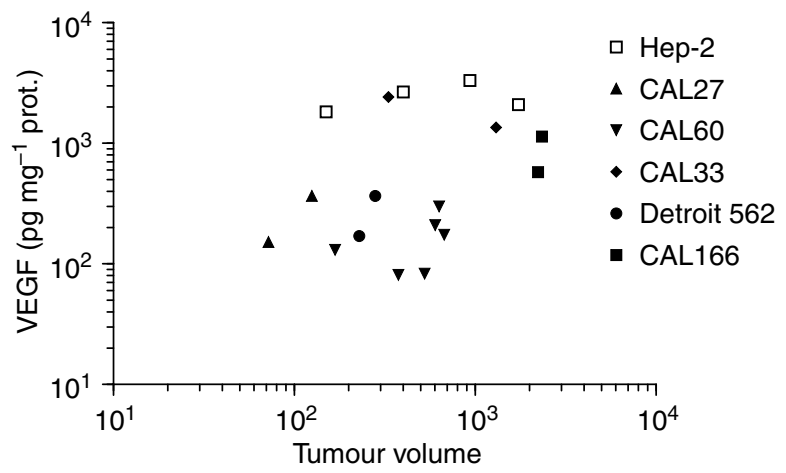

Figure 3 Distribution of individual tumour VEGF levels as a function of tumour volume. A log-log scale was adopted due to the marked variations in the $x$ - and $y$-axis.
(Figure 6). In contrast to what was observed with CD31 labelling, there was no clear link between Ki67 labelling and drug effects (Figure 6). Similar observations were made at the end of the treatment (day 15), but the relative diminution in CD31 labelling resulting from the ZD6126-gefitinib association was less marked at this time than at the end of the experiment on day 33 (data not shown).

\section{DISCUSSION}

Tumour neoangiogenesis is a complex process that involves multiple and interrelated steps dependent on positive and negative regulatory growth factors (Wray et al, 2004). Given its importance in the development of tumour-associated neoangiogenesis (Ferrara, 2005), the VEGF pathway has received constant attention as a target in antiangiogenesis strategies using various approaches (Ferrara, 2004). Among these approaches, one of the most rewarding approach so far at the clinical level is the use of the monoclonal antibody bevacizumab, which acts directly by depleting VEGF levels in physiological fluids (Stern and Herrmann, 2005). Another complementary tumour antiangiogenic strategy consists in developing agents that are able to act directly on the function of established endothelial cells in the tumour (Thorpe et al, 2003). Microtubule-destabilising agents belong to this category, where their mechanism of action is focused on the disruption of the endothelial cell cytoskeleton (Hsieh et al, 2005). One of the drugs belonging to this therapeutic class is ZD6126, a phosphate prodrug of $\mathrm{N}$-acetyl colchinol which has been shown to induce pronounced but reversible changes in immature endothelial cell morphology and to have marked vascular effects and antitumour activity in preclinical models (Davis et al, 2002). Antiangiogenic and vascular disrupting therapies target, at different levels, the expanding tumour endothelial cell network. It was tempting, therefore, to combine these classes of drugs. Such an approach was recently adopted by Siemann and Shi (2004). The authors report that significant antitumour efficacy could be achieved by associating the potent VEGF-R2 and EGFR inhibitor ZD6474 with ZD6126. As endothelial cells express EGFR, some of the global antiangiogenic effects resulting from the use of EGFR-
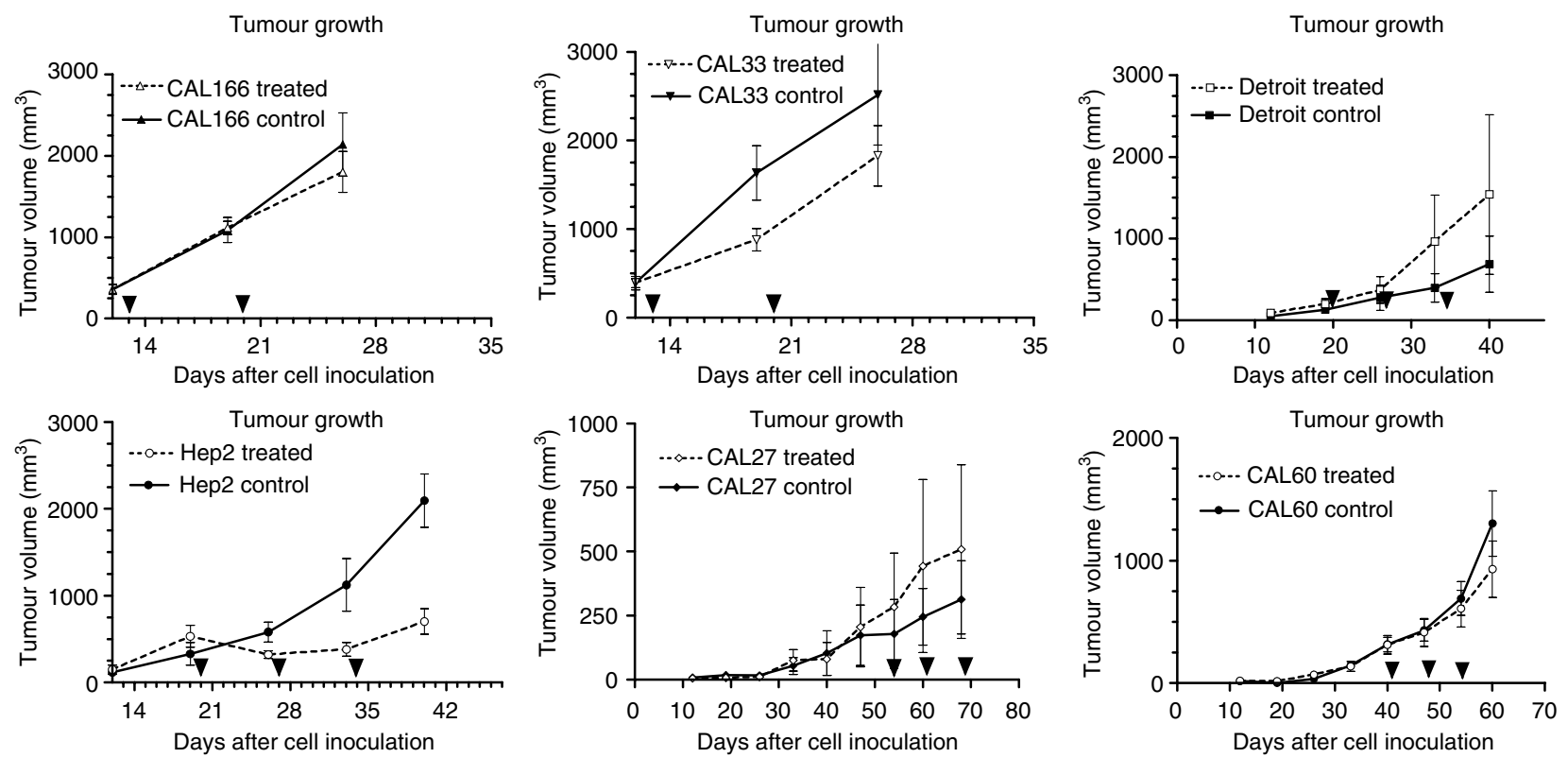

Figure 4 Effects of ZD6126 against CAL33 human head and neck xenografts (filled triangles indicate the time of treatment). Bars represent standard error of the mean. 
A

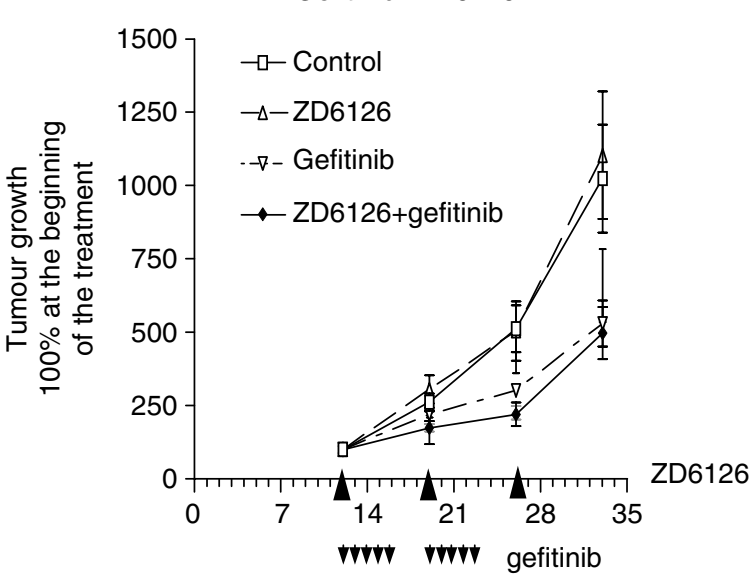

Days after cell inoculation

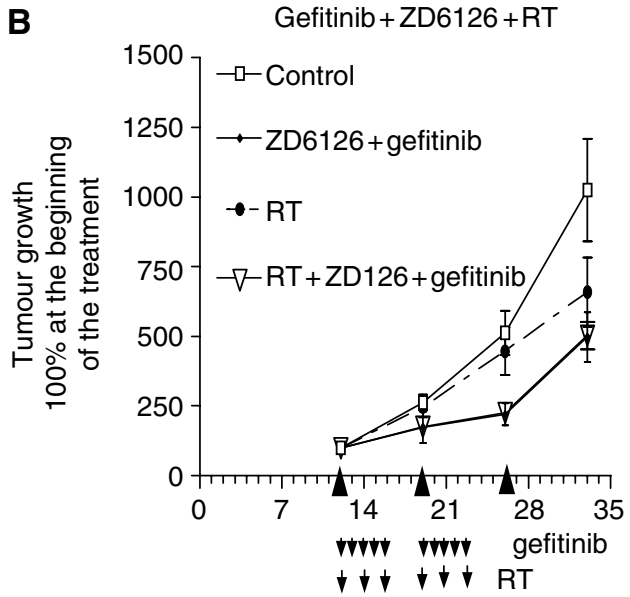

Days after cell inoculation

Figure 5 (A) Effects of ZD6/26 and gefitinib or ZD6/26+ gefitinib on CAL33 tumour xenografts (filled triangles and arrows indicate the time of treatments). Bars represent standard error of the mean. (B) Effects of ZD6126 + gefitinib + RT on CAL33 tumour xenografts (filled triangles and arrows indicate the time of treatments). Tumour growth curves of ZD6126 + gefitinib and ZD6126 + gefitinib + RT were superimposed. Bars represent standard error of the mean.

targeting drugs may also be attributed to the direct impact on endothelial cells (Hirata et al, 2002). Using the same strategy, associating different antiangiogenic approaches, the present study combined the well-known anti-EGFR drug gefitinib with the antivascular agent ZD6126. The study focused on human head and neck cancer cell lines as neoangiogenesis plays a critical role in the development and evolution of tumours arising from the upper aero-digestive tract (Nakaya et al, 2005; Shang and Lir, 2005). Head and neck cancer is also known to express high levels of EGFR with a strong prognostic value, and the 'drugable' relevance of this growth factor has been reported by others (Pomerantz and Grandis, 2003, 2004) and us (Dassonville et al, 1993; Etienne et al, 1999; Pivot et al, 2005).

There is evidence that ZD6126 is active in various preclinical tumour models with variable antitumour efficacy (Blakey et al, 2002; Evelhoch et al, 2004; Skliarenko et al, 2006). One of the objectives of the present investigation was to examine the existence of predictive intra-tumour factors that may explain the observed effects. Based on the study of a panel of six different human cancer
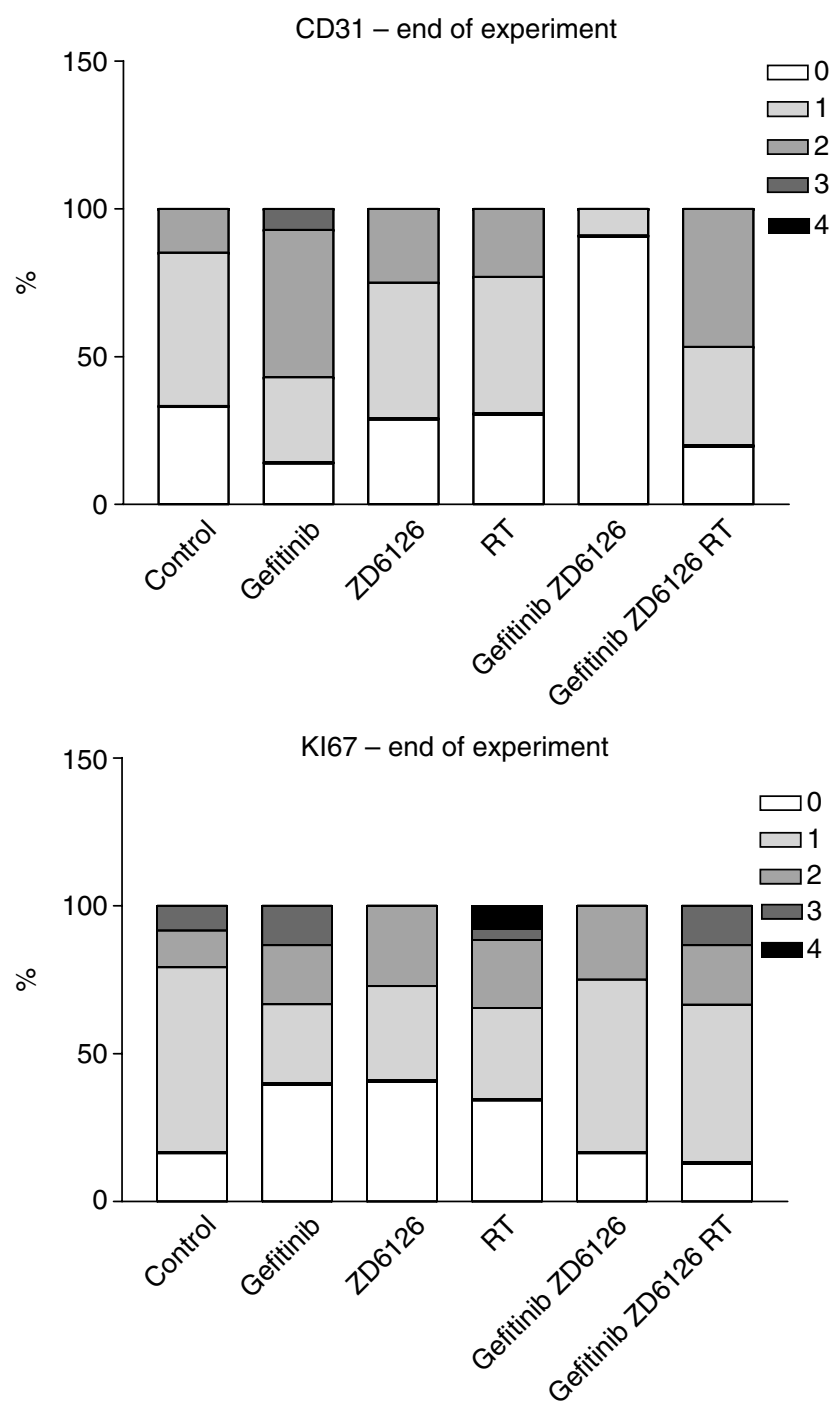

Figure 6 Effects of ZD6126, gefitinib, RT, ZD6|26+ gefitinib and ZD6126 + gefitinib + RT on the labelling frequency and intensity of CD31 (endothelial cell marker) and Ki67 (proliferation marker) in CAL33 tumour xenografts. The final score was the result of the examination of three fields per tumour, and between four and eight tumours were investigated. Labelling intensities are shown as $0=$ no, I = slight, $2=$ medium, $3=$ strong and $4=$ very strong, with the sum of all values in one histogram being 100\%. Scoring was performed at the end of experiments on day 33.

cell lines of head and neck origin, it was confirmed that treatment with ZD6126 results in variable antitumour effects. Of note, from the study by Skliarenko et al (2006), there were tumours for which the application of ZD6126 resulted in greater tumour growth as compared to controls. This phenomenon of tumour re-growth corroborates the present observation of higher growth than in controls for Detroit and CAL27 xenograft under treatment by ZD6126. Antitumour efficacy was observed for CAL33 and Hep-2 cell lines. Interestingly, these two cell lines were those for which established tumours in animals expressed the highest VEGF levels. This result is not particularly surprising and could be explained, as a proof of the concept, by the fact that tumours with a high expression of VEGF may be more dependent on neoangiogenesis and the most sensitive to an antivascular therapeutic approach with ZD6126. Neither EGFR nor IL8 levels were associated with the differences in antitumour effects of ZD6126. The study by Skliarenko et al (2006) put into evidence that tumours with higher 
initial interstitial fluid pressure showed enhanced cell survival following treatment with ZD6126. Thus, intrinsic tumour angiogenesis may be related to the antitumour efficacy of ZD6126. These findings may be useful at the clinical level as there is a risk of a tumour-promoting effect of ZD6126. Selection of appropriate candidates for treatment seems mandatory and could be based on intra-tumour expression of VEGF.

The second part of this study was designed in a way similar to the work recently published by Raben et al (2004) who combined ZD1839 (gefitinib) with ZD6126 and irradiation. The authors reported that the triple association applied to the A549 human non-small-cell lung cancer xenograft model induced the greatest effects on tumour growth and angiogenesis. The conclusions of the present study are somewhat different. First, it is interesting to note that, when examining the gefitinib-ZD6126 association on the CAL33 head and neck human cancer cell line xenograft, it appears that although ZD6126 shows no apparent antitumour efficacy by itself at the dose used in the combination experiment $(150 \mathrm{mg}$ per day), the final effects become supra-additive when combined with gefitinib (Figure 5A). This observation was strengthened by the analysis of the impact of treatment on tumour neoangiogenesis (CD31 labelling). Gefitinib or ZD6126 by themselves had no effect on CD31 tumour labelling compared to controls without drug. In contrast, the combination of these two drugs markedly reduced the intensity of CD31 labelling in the tumours (Figure 6). There was in contrast no evidence for an explanation of the supra-additive effects between the two drugs when examining the impact of combined treatment on tumour intrinsic proliferation capacity (Ki67 labelling). Thus, it seems that the beneficial antitumour effect of associating gefitinib and ZD6126 is more related to the targeting of endothelial cells than to a diminution of the intrinsic tumour growth. The mechanistic explanation for this synergistic effect on tumour angiogenesis may lie in the fact that each drug has a distinctive impact on endothelial cells. ZD6126 directly affects the internal structure of the endothelial cell, whereas gefitinib acts through inhibition of EGFR signalling of endothelial cells and by reduced production of proangiogenic factors by tumour cells (Hirata et al, 2002). The potential direct impact of ZD6126 on the vasculature has been underlined during a recent phase I study with this compound (Beerepoot et al, 2006). Thus, the multiple complementary impacts on endothelial cells may lead to measurable effects on tumour growth, although the effect of ZD6126 alone may not be macroscopically visible at this dose.

Previous experimental studies showed potential beneficial antitumour effects when combining ZD6126 with RT (Siemann and Rojiani, 2002; Raben et al, 2004). A recent study (Wachsberger et al, 2005), however, drew more contrasting conclusions with data suggesting that the optimal therapeutic benefit of ZD6126 plus RT (U87 glioblastoma xenograft) is schedule-dependent with combinations of ZD6126 before each dose of RT being less effective than RT alone. The importance of the scheduling involving ZD6126 and RT was also reported by a previous study (Siemann and Rojiani, 2002) that showed that this association increased tumour cell killing of KHT mouse sarcoma when given $24 \mathrm{~h}$ before RT or $1 \mathrm{~h}$ or more following RT, but was not found to be as effective if given $1 \mathrm{~h}$ before RT. In the present study, the sequence of association between RT and ZD6126 was taken into consideration as the drug was given $2 \mathrm{~h}$ after RT. The impact of the association of RT with drugs was examined with the triple combination and it was found, in the present conditions, that RT did not markedly modify the antitumour effect resulting from the ZD6126-gefitinib association (Figure 5B). Of note, this observation was corroborated by examining CD31 tumour labelling, which showed a less intense impact on this parameter when applying the triple combination as compared to the gefitinib-ZD6126 association.

Overall, the present study may contribute to future innovative clinical applications and suggests that the VEGF tumour level is a possible predictor for ZD6126 antitumour efficacy, that it strengthens the notion of antitumour supra-additive effects when combining gefitinib and ZD6126, identifies neoangiogenesis as the main impact of this synergistic combination and gives no firm support to the benefit of adding RT to the drug association.

\section{REFERENCES}

Beerepoot LV, Radema SA, Witteveen EO, Thomas T, Wheeler C, Kempin S, Voest EE (2006) Phase I evaluation of weekly administration of the novel vascular-targeting agent, ZD6126, in patients with solid tumors. J Clin Oncol 24: $1491-1498$

Blakey DC, Westwood FR, Walker M, Hughes GD, Davis PD, Ashton SE, Ryan AJ (2002) Antitumor activity of the novel vascular targeting agent ZD6126 in a panel of tumor models. Clin Cancer Res 8: 1974-1983

Ciardiello F, Caputo R, Bianco R, Damiano V, Fontanini G, Cuccato S, De Placido S, Bianco AR, Tortora G (2001) Inhibition of growth factor production and angiogenesis in human cancer cells by ZD1839 (Iressa), a selective epidermal growth factor receptor tyrosine kinase inhibitor. Clin Cancer Res 7: 1459-1465

Dassonville O, Formento JL, Francoual M (1993) Expression of epidermal growth factor receptor and survival in upper aerodigestive tract cancer. J Clin Oncol 11: 1873-1878

Davis PD, Dougherty GJ, Blakey DC, Galbraith SM, Tozer GM, Holder AL, Naylor MA, Nolan J, Stratford MR, Chaplin DJ, Hill SA (2002) ZD6126: a novel vascular-targeting agent that causes selective destruction of tumor vasculature. Cancer Res 62: $7247-7253$

Etienne MC, Pivot W, Formento JL, Bensadoun RJ, Formento P, Dassonville O, Francoual M, Poissonnet G, Fontana X, Schneider M, Demard F, Milano G (1999) A multifactorial approach including tumoural epidermal growth factor receptor, p53, thymidylate synthase and dihydropyrimidine dehydrogenase to predict treatment outcome in head and neck cancer patients receiving 5-fluorouracil. $\mathrm{Br} J$ Cancer 79: $1864-1869$

Evelhoch JL, LoRusso PM, He Z, DelProposto Z, Polin L, Corbett TH, Langmuir P, Wheeler C, Stone A, Leadbetter J, Ryan AJ, Blakey DC, Waterton JC (2004) Magnetic resonance imaging measurements of the response of murine and human tumors to the vascular-targeting agent ZD6126. Clin Cancer Res 10: $3650-3657$

Ferrara N (2004) Vascular endothelial growth factor as a target for anticancer therapy. Oncologist 9: 2-10

Ferrara N (2005) The role of VEGF in the regulation of physiological and pathological angiogenesis. EXS 94: 209-231

Formento P, Hannoun-Levi JM, Gérard F, Mazeau C, Fischel JL, EtienneGrimaldi MC, Gugenheim J, Milano G (2005) Gefitinib-trastuzumab combination on hormone-refractory prostate cancer xenograft. Eur J Cancer 41: $1467-1473$

Goto H, Yano S, Matsumori Y, Ogawa H, Blakey DC, Sone S (2004) Sensitization of tumor-associated endothelial cell apoptosis by the novel vascular-targeting agent ZD6126 in combination with cisplatin. Clin Cancer Res 10: $7671-7676$

Gross ME, Shazer RL, Agus DB (2004) Targeting the HER-kinase axis in cancer. Semin Oncol 31: 9-20

Hirata A, Ogawa S, Kometani T, Kuwano T, Naito S, Kuwano M, Ono M (2002) ZD1839 (Iressa) induces antiangiogenic effects through inhibition of epidermal growth factor receptor tyrosine kinase. Cancer Res 62: $2554-2560$

Hsieh HP, Liou JP, Mahindroo N (2005) Pharmaceutical design of antimitotic agents based on combretastatins. Curr Pharm Des 11: $1655-1677$

Le QT, Giaccia AJ (2003) Therapeutic exploitation of the physiological and molecular genetic alterations in head and neck cancer. Clin Cancer Res 9: $4287-4295$

Magné N, Fischel JL, Dubreuil A, Formento P, Marcié S, Lagrange JL, Milano G (2002) Sequence-dependent effects of gefitinib (Iressa) in combination with cytotoxic treatment in human head and neck cancer. Br J Cancer 86: 819-827

Nakaya H, Kawashiri S, Tanaka A, Noguchi N, Kato K, Hase T, Yamamoto $\mathrm{E}$ (2005) Influences of angiogenesis and lymphangiogenesis on cancerous 
invasion in experimentally induced tongue carcinoma. J Oral Pathol Med 34: $87-92$

Perrotte P, Matsumoto T, Inoue K, Kuniyasu H, Eve BY, Hicklin DJ, Radinsky R, Dinney CP (1999) Anti-epidermal growth factor receptor antibody C225 inhibits angiogenesis in human transitional cell carcinoma growing orthotopically in nude mice. Clin Cancer Res 2: $257-265$

Pivot X, Magné N, Guardiola E, Poissonnet G, Dassonville O, Francoual M, Formento JL, Bensadoun RJ, Demard F, Schneider M, Milano G (2005) Prognostic impact of the epidermal growth factor receptor levels for patients with larynx and hypopharynx cancer. Oral Oncol 41: 320-327

Pomerantz RG, Grandis JR (2003) The role of epidermal growth factor receptor in head and neck squamous cell carcinoma. Curr Oncol Rep 5: $140-146$

Pomerantz RG, Grandis JR (2004) The epidermal growth factor receptor signaling network in head and neck carcinogenesis and implications for targeted therapy. Semin Oncol 31: 734-743

Prewett MC, Hooper AT, Bassi R (2002) Enhanced antitumor activity of anti-epidermal growth factor receptor monoclonal antibody IMC-C225 in combination with irinotecan (CPT-11) against human colorectal tumor xenografts. Clin Cancer Res 8: 994-1003

Raben D, Bianco C, Damiano V, Bianco R, Melisi D, Mignogna C, D’Armiento FP, Cionini L, Bianco AR, Tortora G, Ciardiello F, Bunn P (2004) Antitumor activity of ZD6126, a novel vascular-targeting agent, is enhanced when combined with ZD1839, an epidermal growth factor receptor tyrosine kinase inhibitor, and potentiates the effects of radiation in a human non-small cell lung cancer xenograft model. Mol Cancer Ther 3: $977-983$

Shang ZJ, Lir JR (2005) Expression of endothelial nitric oxide synthase and vascular endothelial growth factor in oral squamous cell carcinoma: its correlation with angiogenesis and disease progression. J Oral Pathol Med 34: $134-139$

Siemann DW, Rojiani AM (2002) Enhancement of radiation therapy by the novel vascular targeting agent ZD6126. Int J Radiat Oncol Biol Phys 53: $164-171$

Siemann DW, Shi W (2004) Efficacy of combined antiangiogenic and vascular disrupting agents in treatment of solid tumors. Int J Radiat Oncol Biol Phys 60: 1233-1240

Simon R, Mirlacher M, Sauter G (2004) Tissue microarrays. Biotechniques 36: $98-105$

Skliarenko JV, Lunt SJ, Gordon ML, Vitkin A, Milosevic M, Hill RP (2006) Effects if the vascular disrupting agent ZD6126 on interstitial fluid pressure and cell survival in tumors. Cancer Res 66: 2074-2080

Stern M, Herrmann R (2005) Overview of monoclonal antibodies in cancer therapy: present and promise. Crit Rev Oncol Hematol 54: 11-29

Thorpe PE, Chaplin DJ, Blakey D (2003) The first international conference on vascular targeting: meeting overview. Cancer Res 63: 1144-1147

Wachsberger P, Burd R, Marero N, Daskalakis C, Ryan A, McCue P, Dicker AP (2005) Effect of the tumor vascular-damaging agent, ZD6126, on the radioresponse of U87 glioblastoma. Clin Cancer Res 11: $835-842$

Woodburn JR (1999) The epidermal growth factor receptor and its inhibition in cancer therapy. Pharmacol Ther 82: 241-250

Workman P, Twentyman P, Balkwill F, Balmain A, Chaplin D, Double J, Embleton J, Newell D, Raymond R, Stables J, Stephens T, Wallace J (1998) United Kingdom Co-ordinating Committee on Cancer Research (UKCCCR) guidelines for the welfare of animals in experimental neoplasia (second edition). Br J Cancer 77: 1 - 10

Wray CJ, Rilo HL, Ahmad SA (2004) Colon cancer angiogenesis and antiangiogenic therapy. Expert Opin Investig Drugs 13: 631-641 